\title{
Editorial
}

\section{Tribology of the Head-Disk Interface}

\author{
Bruno Marchon, ${ }^{1}$ Norio Tagawa, ${ }^{2}$ Bo Liu, ${ }^{3}$ Tom Karis, ${ }^{1}$ and Jia-Yang Juang ${ }^{4}$ \\ ${ }^{1}$ HGST, San Jose Research Center, 3403 Yerba Buena Road, San Jose, CA 95135, USA \\ ${ }^{2}$ Department of Mechanical Engineering, Faculty of Engineering Science, Kansai University, 3-3-35 Yamate-cho, Suita, \\ Osaka 564-8680, Japan \\ ${ }^{3}$ Data Storage Institute, Agency for Science, Engineering and Research, DSI Building, 5 Engineering Drive, Singapore 117608 \\ ${ }^{4}$ Department of Mechanical Engineering, National Taiwan University, 713 Engineering Building, No. 1, Section 4, Roosevelt Road, \\ Taipei 10617, Taiwan
}

Correspondence should be addressed to Bruno Marchon; bruno.marchon@hgst.com

Received 3 March 2013; Accepted 3 March 2013

Copyright (C) 2013 Bruno Marchon et al. This is an open access article distributed under the Creative Commons Attribution License, which permits unrestricted use, distribution, and reproduction in any medium, provided the original work is properly cited.

Hard Disk Drives (HDD) are perhaps the unsung heroes of today's information technology. The rise and growth of the Internet search engines, social media, and cloud computing have been enabled, in part, by the inexpensive and massive storage capabilities that HDD technology provides. Less than 60 years after its invention by IBM in San Jose in 1956, the hard disk drive has seen an unrelentless, Moore's Law-like growth in storage density, as well as a concomitant decrease in price and form factor. The first HDD ever commercialized, IBM's RAMAC, had a mere 5 megabytes of storage, cost over a million dollar in today's currency, and weighted over a ton. Today's largest capacity disk drives hold 4 terabytes of data in a small 3.5 inch form factor and at a price similar to that of small household appliances such as a coffee maker, a hair dryer, or a toaster.

Unlike other components of modern computers, the disk drive has moving parts, with a read/write head flying over a rotating disk. The thickness of the air gap between the flying head and the disk is now of the order of a nanometer or so, for velocities greater than $10 \mathrm{~m} / \mathrm{s}$, thus making the headdisk interface (HDI) one of the most advanced tribological systems available to the consumer in today's marketplace. As a result, great advances have been made over the last few decades in the field of nanotribology (the tribology of nanoscale systems), both in a fundamental, and an applied point of view.

This special issue contains 10 papers of great interest to the field, touching on an array of relevant topics, and research papers as well as review papers. A useful backdrop to this issue is a review of the HDI roadmap to an areal density of 4 terabit-per-square inch, discussed by researchers from the two largest HDD companies. Three papers attempt to describe and model the frictional behavior of head-disk contacts when they occur. H. Tani et al. present experimental friction data from a pin rubbing against a disk and discuss how it is affected by the roughness of the contact as well as the chemical nature of the lubricant end-group functionalities. S. Itoh et al. use a "fiber wobbling" method to understand the viscoelastic properties of the lubricant film under confinement. They provide evidence of a transition from a viscous to a viscoelastic behavior when the film becomes molecularly thin. Finally, P. Jones et al. present some molecular dynamics simulations of two hydrogen-terminated diamond surfaces in sliding contact and demonstrate a JKR/DMT-like behavior at the molecular scale.

As far as the dynamics of the flying head is concerned, S. Canchi et al. discuss the behavior of the thermally actuated head when it contacts the disk, a topic of great importance as such a scheme is used in all disk drives today in order to calibrate the head-to-disk clearance. K. S. Myo et al., on the other hand, elaborate on how the use of Heat-Assisted Magnetic Recording (HAMR) will affect the head air-bearing characteristics.

On the topic of disk lubricant, R. Waltman and H. Deng offer new evidence that using a low molecular weight disk lubricant enhances head-disk clearance, owing to energetic considerations, but perhaps at the expense of small evaporation losses. Furthermore, M. S. Jhon et al. present a review of 
multiscale molecular dynamics, allowing the modeling and prediction of the lubricant behavior and spanning orders of magnitude in time and length from nano- to meso- and to macroscales. Finally, the issue of HDD reliability is also addressed with a paper by J. He that describes a novel instrumentation for nanoscale disk defect detection and characterization, as well as a paper by K. Sonoda discussing how organic contamination affects the dynamics of the flying head.

We would like to thank the authors of all these papers for publishing such a valuable set of papers that provide an important scientific backdrop to the state of the head-disk interface technology in today's disk drives. We hope that this issue will stimulate further fundamental insight in this topic, to enable the HDD industry to keep delivering highperformance storage solutions to our digital world.

Bruno Marchon Norio Tagawa

Bo Liu

Tom Karis

Jia-Yang Juang 

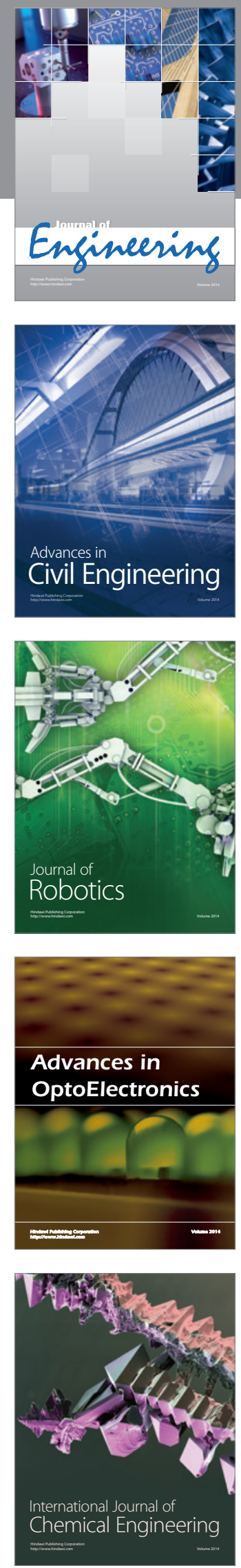

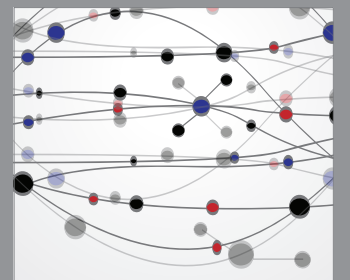

The Scientific World Journal
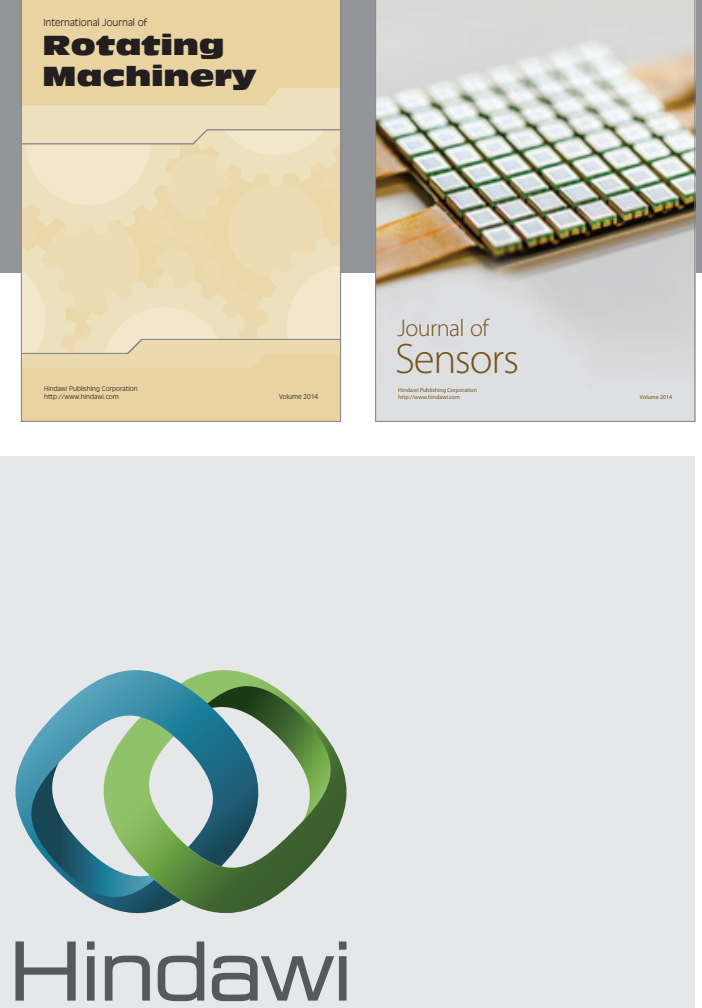

Submit your manuscripts at http://www.hindawi.com
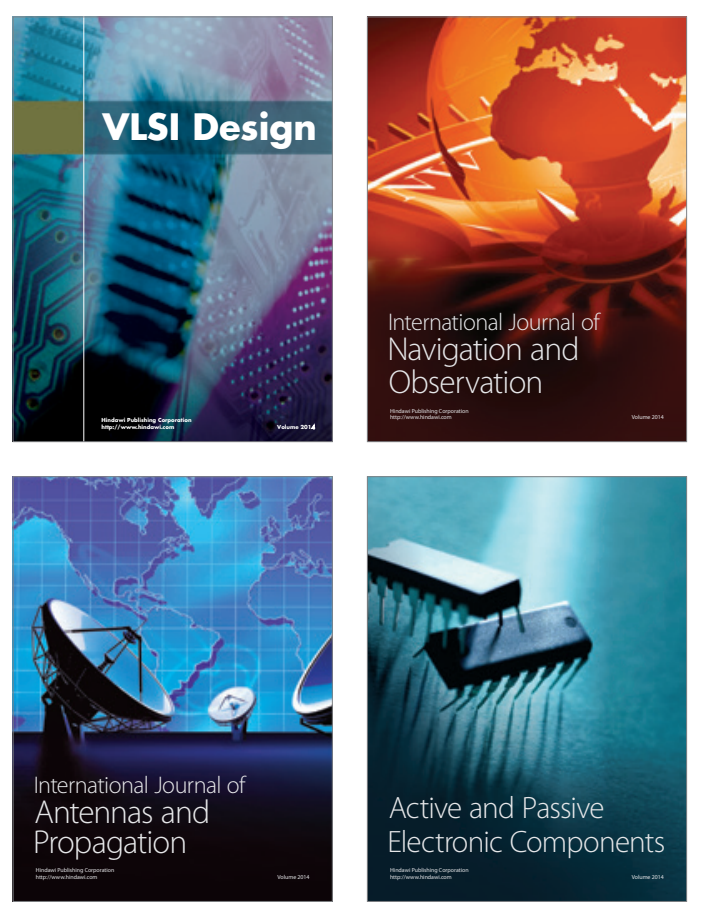
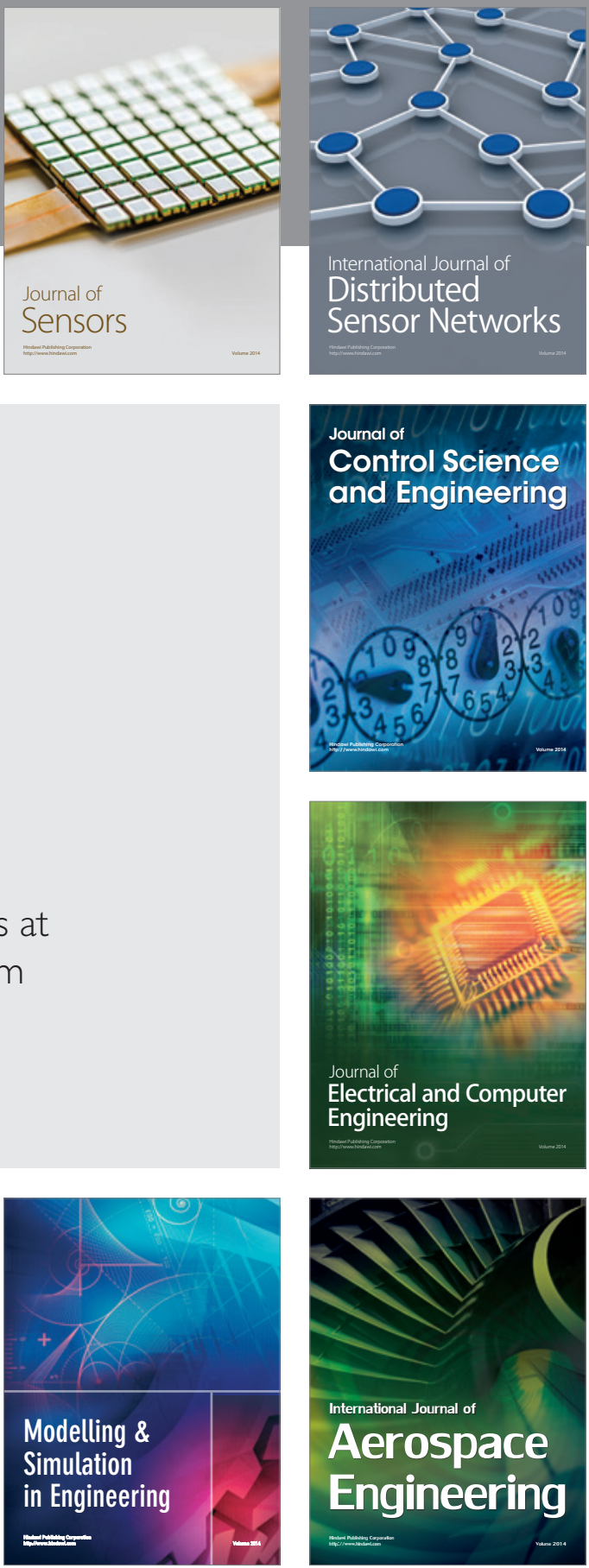

Journal of

Control Science

and Engineering
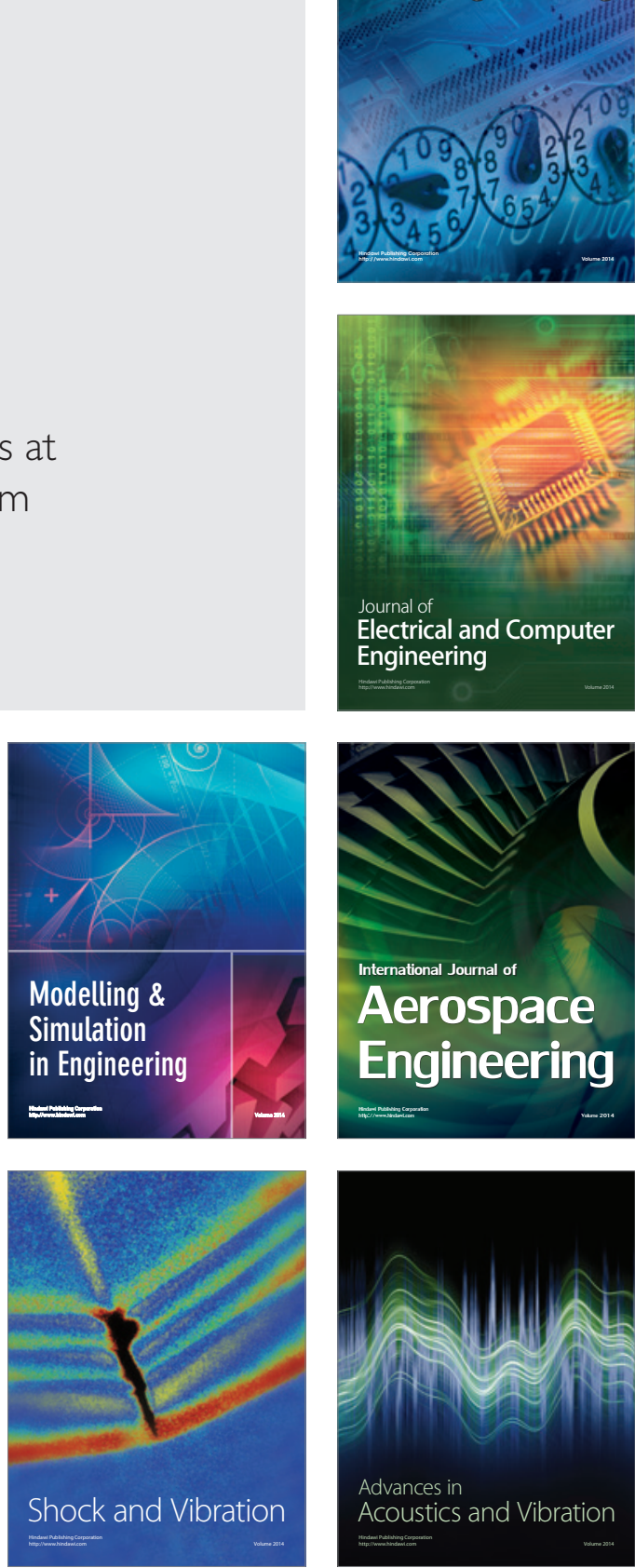